\title{
Numerical modelling in non linear fracture mechanics
}

( da ESIS Newsletter 2005)

\author{
Viggo Tvergaard \\ Dept. of Mechanical Engineering, Solid mechanics, Technical University of Denmark, Nils Koppels Allé, \\ Building 404, DK-2800 Kgs. Lyngby, Denmark
}

\begin{abstract}
Some numerical studies of crack propagation are based on using constitutive models that account for damage evolution in the material. When a critical damage value has been reached in a material point, it is natural to assume that this point has no more carrying capacity, as is done numerically in the element vanish technique. In the present review this procedure is illustrated for micromechanically based material models, such as a ductile failure model that accounts for the nucleation and growth of voids to coalescence, and a model for intergranular creep failure with diffusive growth of grain boundary cavities leading to micro-crack formation. The procedure is also illustrated for low cycle fatigue, based on continuum damage mechanics. In addition, the possibility of crack growth predictions for elastic-plastic solids using cohesive zone models to represent the fracture process is discussed.
\end{abstract}

KEYWORDS: Damage evolution, crack growth, coesive zone

\section{INTRODUCTION}

Many procedures for the analysis of crack propagation are based on using critical values of parameters characterising the crack-tip stress and strain fields, such as the stress intensity factor, the J-integral, the crack-tip opening displacement, or the crack-tip opening angle. Alternatively, the prediction of crack growth may be directly based on the fracture mechanism operating on the microscale, either by incorporating the failure mechanism in the constitutive equations for the material, or by representing the failure mechanism through a cohesive zone model of the fracture process zone. The present paper will give a survey of a number of investigations where the prediction of crack growth has been based on models of the actual fracture mechanism.

One of the most well known material models that accounts for the micromechanics of damage is the modified Gurson model [1,2], which models the evolution of ductile fracture by the nucleation and growth of voids to coalescence. Some of the analyses using this model to predict ductile crack growth will be discussed. Also for creep failure in metals at high temperatures material models [3] have incorporated the micromechanisms of diffusive cavity growth in grain boundaries, leading to open micro-cracks at grain boundary facets at a rate strongly affected by grain boundary sliding. Results on creep crack growth based on this failure model will be mentioned. The term continuum damage mechanics is used for constitutive relations, which are able to represent the effect of damage evolution on the macro level, by developing appropriate expressions in which free material parameters can be fitted to experiments, as in the case of low cycle fatigue [4]. As an example, predictions of mi- cro-crack formation in a metal matrix composite, based on this material model, will be presented here.

Cohesive zone models have been used in recent years in a number of analyses of crack growth resistance in elasticplastic solids [5]. Some of the predictions obtained in these studies will be briefly mentioned here.

\section{MATERIAL MODELS WITH DAMAGE EVOLUTION}

When the failure mechanism is incorporated in in the constitutive relations, the crack growth follows directly from the predicted loss of stress carrying capacity in one or more integration points in an element. Then it is natural to kill the failed elements, by using the element vanish technique [6]. This procedure has been used for the predictions of crack growth to be discussed in the following three subsections.

\section{Crack growth by ductile failure}

Much interest has been devoted to the development of elastic-plastic or viscoplastic constitutive equations that account for the effect of ductile damage development. The most well known model is that suggested by Gurson [1], which makes use of an approximate yield condition $\Phi\left(\sigma^{i j}, \sigma_{M}, f\right)=0$ for a material containing a volume fraction $\mathrm{f}$ of voids, where $\sigma^{i j}$ is the average macroscopic Cauchy stress tensor and $\sigma_{M}$ is an equivalent tensile flow stress representing the actual microscopic stress-state in the matrix material. With some modifications to improve predictions of plastic flow localization 
[7] and of final failure by void coalescence [8] this yield condition is of the form

$\Phi=\frac{\sigma_{e}^{2}}{\sigma_{M}^{2}}+2 q_{1} f^{*} \cosh \left(\frac{q_{2}}{2} \frac{\sigma_{k}^{k}}{\sigma_{M}}\right)-\left[1+\left(q_{1} f^{*}\right)^{2}\right]=0$

where $\sigma_{e}=\left(3 s_{i j} s^{i j} / 2\right)^{1 / 2}$ is the macroscopic effective Mises stress, and $s^{i j}=\sigma^{i j}-G^{i j} \sigma_{k}^{k} / 3$ is the stress deviator. This material model accounts for the growth of the void volume fraction $f$ due to plastic flow of the material around voids and due to the nucleation of new voids, and final failure is directly predicted when $f$ reaches the critical value, at which the yield surface has shrunk to a point.

This material model has been applied in a number of numerical studies of crack growth, including some studies where two populations of void nucleating particles are modelled; large weak particles that nucleate voids at relatively small strains and small strong particles that nucleate voids at much larger strains. For an edge cracked specimen under dynamic loading [9] results of a plane strain analysis are shown in Fig. 1, where contours of constant void volume fraction define the predicted crack growth path in a case of a random distribution of the larger inclusions ahead of the initial crack-tip.

Also a full three dimensional analysis has been used to analyse this type of specimen [10]. Here the computer requirements were much larger, but the advantage is that more realistic spherical shapes of the larger inclusions can be accounted for, and that 3D modes of growth are accounted for, such as tunnelling and shear lip formation. Continuations of the 3D fracture study have been carried out recently in analyses that do not directly focus on crack growth, e.g. the failure of a metal matrix composite [11] or of a Charpy V-notch specimen cut through a weld [12]. Some attempts to include a damage dependent material length scale in this constitutive model have been carried out by Leblond et al. [13] and Tvergaard and Needleman [14], using an integral condition on the rate of increase of the void volume fraction.

The expressions used in [14] are

$$
\begin{gathered}
\dot{f}\left(y^{i}\right)=\frac{1}{W\left(y^{i}\right)} \int_{V} \dot{f}_{\text {local }}\left(\hat{\mathrm{y}}^{\mathrm{i}}\right) w\left(y^{i}-\hat{y}^{i}\right) d \hat{V} \\
w\left(y^{i}\right)=\left[\frac{1}{1+(z / L)^{p}}\right]^{q}, w\left(y^{i}\right)=\int_{V} w\left(y^{i}-\hat{y}^{i}\right) d \hat{V}
\end{gathered}
$$

where $L>0$ is the material characteristic length, $z=\sqrt{g_{i j} y^{i} y^{j}}$, and $p=8, q=2$. The usual local formulation corresponds to the limit $L \rightarrow 0$, and it has been shown, as for other non-local continuum models, that the mesh dependence of numerical solutions in a softening regime are removed by taking $L>0$. This nonlocal damage model has been applied by Needleman and Tver- gaard [15] to predict ductile crack growth in the edge cracked specimen under dynamic loading also analysed in $[9,10]$.

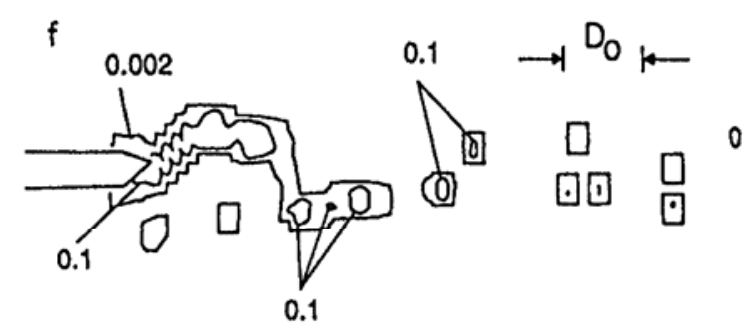

(a)

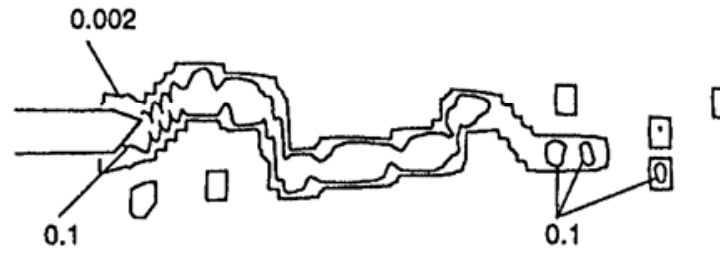

(b)

Figure 1: Crack growth indicated by contours of constant void volume fraction, $\mathrm{f}$, for random distribution of larger particles.

(a) $t=1.5 \mu \mathrm{s}, \quad \Delta a=0.09 \mathrm{~mm}$;

(b) $t=1.6 \mu \mathrm{s}, \quad \Delta a=0.27 \mathrm{~mm}$. (From [9]).

\section{Creep crack growth}

High temperature failure leading to crack growth has been modelled in terms of continuum damage mechanics (Hayhurst et al. [16]), where damage parameters are fitted to material behaviour on the macro level. The micromechanisms of creep failure in polycrystalline metals involve the nucleation and growth of small voids to coalescence; but here diffusion plays an important role, and the cavities occur primarily on grain boundary facets perpendicular to the maximum principal tensile stress (e.g. Ashby and Dyson [17]), where a creep constraint on the rate of cavitation is often a dominant mechanism. Cavity coalescence on a grain boundary facet leads to a microcrack, and final intergranular failure occurs as such micro-cracks link up. Grain boundary sliding is an important mechanism that further complicates the analysis of creep failure. A micromechanically based constitutive model for creep failure in a polycrystalline metal has been proposed (Tvergaard $[3,18]$ ), in which the macroscopic creep strain rate is given by the expression

$$
\begin{gathered}
\dot{\eta}_{i j}^{C}=\dot{\varepsilon}_{0}\left(1+C \varepsilon_{e}^{C}\right)\left(\frac{\sigma_{e}}{\sigma_{0}}\right)^{n}\left[\frac{3}{2} \frac{s_{i j}}{\sigma_{e}}\left(f^{*}\right)^{n}+\right. \\
\left.\rho^{*}\left\{\frac{3}{2} \frac{n-1}{n+1} \frac{s_{i j}}{\sigma_{e}}\left(\frac{S^{*}-\sigma_{n}}{\sigma_{e}}\right)^{2}+\frac{2}{n+1} \frac{S^{*}-\sigma_{n}}{\sigma_{e}} m_{i j}^{*}\right\}\right]
\end{gathered}
$$

Here, $n$ is the creep power, $C>0$ represents substructure induced acceleration of creep, and expressions for other parameters are determined by axisymmetric cell 
model studies for a grain with a cavitating facet and sliding boundaries [3]. If there is no sliding, $f^{*}$ is unity, $\rho^{*}$ is the density of cavitating facets $m_{i j}^{*}$ is a direction tensor for cavitating facets, and $S^{*}-\sigma_{n}$ is the difference between the maximum principal stress and the normal stress on a cavitating facet. The material model has been used to predict crack growth [18], by applying the element vanish technique when cavity coalescence was predicted on a grain boundary. For a double edge cracked panel under tension Fig. 2 shows the predicted damage near the crack-tip at two stages of time, where the damage parameter $a / b$ is the cavity radius divided by the cavity half spacing on a facet, and vanished triangular elements are painted black.

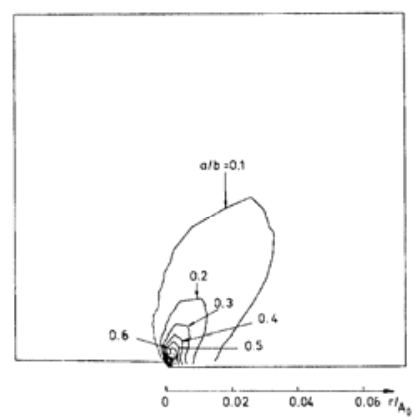

(a)

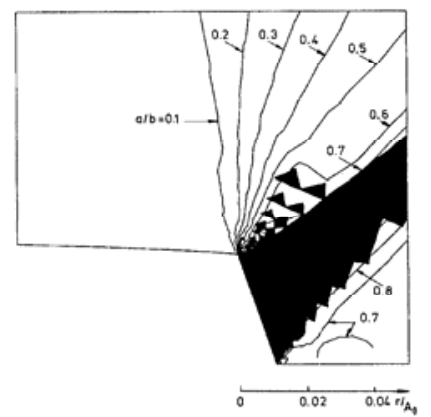

(b)
Figure 2: Distributions of creep damage ahead of a crack-tip. Continuous cavity nucleation, no grain boundary sliding, and $C=40$. (a) $t / t_{f}^{0}=0.064$. (b) $t / t_{f}^{0}=0.686$. (From [18]).

Plane strain multi-grain cell models for a polycrystalline aggregate have been used by van der Giessen and Tvergaard [19] to study the final creep fracture process, as microcracks formed at grain boundary facets link up. Such analyses are however limited by the unrealistic grain geometry and the reduced constraint on sliding. But a great advantage is that large grain arrays can be analysed if a crude mesh is used within each grain, and this allows for direct modelling of intergranular crack growth in a plane strain multi-grain aggregate (Onck and van der Giessen [20]).

\section{Fatigue cracking}

Among the many applications of continuum damage mechanics [4], studies of failure by low cycle fatigue are an important example, where a material model directly based on the micro mechanics of failure has not been developed. As the development of fatigue fracture depends strongly on the plastic strain range in each cycle, an accurate cyclic plasticity model is needed (e.g. Ohno and Wang [21]), with damage mechanics incorporated. The scalar damage parameter $D$ is taken to be zero initially, but when the accumulated plastic strain $p$ reaches a threshold value $p_{d}$, it is assumed that damage starts to develop according to the evolution law

$$
\dot{D}=\frac{Y}{S} \dot{p} \alpha(p), \alpha=\left\{\begin{array}{lll}
1, & \text { if } & p \geq p_{d} \\
0, & \text { if } & p<p_{d}
\end{array}\right.
$$

Here, $S$ is a material parameter describing the energy strength of damage, the strain energy release rate is given by $Y=\sigma_{e}^{2} R_{V} /\left(2 E(1-D)^{2}\right)$, and the expression for $R_{V}$ depends on the mean stress $\sigma_{k k} / 3$, so that fatigue develops more rapidly under tensile stresses. When the damage parameter reaches a critical value $D_{c}$, this is taken to represent such a high density of microcracks that coalescence into a macrocrack occurs. In a finite element analysis this failure event is represented in terms of the element vanish technique, such that the model can be used to predict the growth of a macroscopic crack. This type of numerical study has been carried out in [22] for a metal matrix composite, where the fatigue crack growth occurs in the metal matrix around short brittle fibres.

\section{MODELLING BY COESIVE ZONE}

As an alternative to the continuum models discussed above, a number of crack growth analyses describe the fracture process separately in terms of a traction separation law for the crack surface, while the inelastic deformations around the crack are accounted for by standard plasticity without damage. This gives an attractive possibility for separating effects of fracture process parameters from effects of the material parameters determining inelastic deformations, e.g. in relation to determining crack growth resistance curves. Thus, analyses of this type determine directly the ratio between the remote fracture toughness and the local fracture toughness determined by the assumed cohesive model.

In [5] a rather general case of crack growth along the interface between an elastic-plastic solid and a rigid solid was studied. Here, a cohesive zone model was needed that accounts for both normal and tangential separation, or mixtures of these, not only in order to study effects of remote mixed mode loading, but also because of the oscillating elastic singularity resulting from the elastic mismatch across the interface, which gives varying mixtures of normal stress and shear stress along the interface. This work has been continued in a number of different studies of interface debonding, for different types of material systems. Thus, in [23] resistance curves have been determined numerically for crack growth along an interface joining two elastic-plastic solids, or an elastic-plastic solid to an elastic substrate. The steady-state value $|K|_{\text {ss }}$ of the remote fracture toughness is found when the resistance curves reach their maximum, which depends on the local mode mixity $\psi_{0}$ near the crack-tip. As an example Fig. 3 shows such steady-state values for a case with an elastic substrate, where the elastic modulus $E_{2}$ in the substrate is twice that in the elastic-plastic solid. The angular measure $\psi_{0}$ is near $0^{\circ}$ for mode I loading and would be near $90^{\circ}$ or $-90^{\circ}$ for mode II loading. The steady-state toughnesses are normalised by the value $K_{0}$ corresponding to a purely elastic solid, for the separation 
energy assumed in the traction separation law. The different curves correspond to different values of the peak stress $\hat{\sigma}$ for the traction separation law, normalised by the initial yield stress. The curves show two typical features of such results, that the fracture toughness level is very sensitive to small increases of the peak stress, and that the curves have minima for near mode I conditions at the crack-tip.

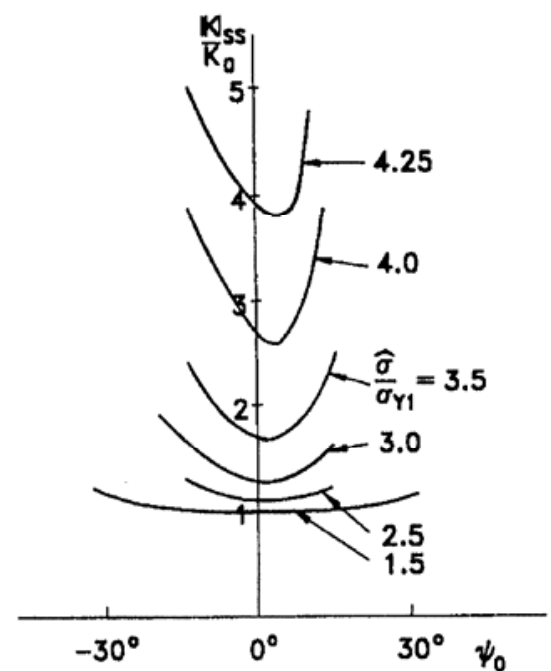

Figure 3: Steady-state interface toughness as a function of the local mixity measure $\psi_{0}$, for $\sigma_{Y 1} / E_{1}=0.003$ and $\sigma_{Y 2} \square \infty$, considering different values of $\sigma / \sigma_{Y 1}, E_{2} / E_{1}=2$. (From [23]).

\section{REFERENCES}

[1] A.L. Gurson, Engng. Mater. Technol., 99 (1977) 2.

[2] V. Tvergaard, Advances in Applied Mechanics, Academic Press, Inc., 27 (1990) 83.

[3] V. Tvergaard, Acta Metallurgica, 32 (1984) 1977.

[4] J. Lemaitre, A Course on Damage Mechanics. Springer-Verlag, (1992).

[5] V. Tvergaard, J.W. Hutchinson, J. Mech. Phys. Solids, 41 (1993) 1119.

[6] V. Tvergaard, J. Mech. Phys. Solids, 30 (1982) 399.

[7] V. Tvergaard, Int. J. Fracture, 17 (1981) 389.

[8] V.Tvergaard, A.Needleman, Acta Metall., 32 (1984) 157.

[9] V. Tvergaard, A. Needleman, J. Mech. Phys. Solids, 40 (1992) 447.

[10] K.K. Mathur, A. Needleman, V. Tvergaard, J. Mech. Phys. Solids, 44 (1996) 439.

[11] V. Tvergaard, Modelling Simul. Mater. Sci. Eng., 9 (2001) 143.

[12] V. Tvergaard, A.Needleman, 3D Charpy Specimen Analyses for Welds, to appear in Proc. Charpy Centenery Conf. (2001).

[13] J.B. Leblond, G. Perrin, J. Devaux, J. Appl. Mech. 61 (1994) 236.

[14] V. Tvergaard, A. Needleman, Int. J. Solids Structures 32 (1995) 1063.
[15] A. Needleman, V.Tvergaard, Eur. J. Mech., A/Solids, 17 (1998) 421.

[16] D.R. Hayhurst, P.R.Brown, C.J. Morrison, Philosophical Transactions, Royal Society London A311 (1984) 131.

[17] M.F. Ashby, B.F. Dyson, National Physical Laboratory, Report DMA(A), (1984) 77.

[18] V. Tvergaard, Int. J. Fracture, 42 (1990) 145.

[19] E. van der Giessen, V. Tvergaard, Acta Metall. Mater., 42 (1994) 959.

[20] P.R. Onck, E. van der Giessen, Mech. Mater, 26 (1997) 109.

[21] N. Ohno, J.-D. Wang, Int. J. of Plasticity, 9 (1993) 375.

[22] V. Tvergaard, T.Ø. Pedersen, Arch. Mech., 52 (2000) 799.

[23] V. Tvergaard, J. Mech. Phys. Solids, 49 (2001) 2689. 